\title{
A SUSTENTABILIDADE E A ECONOMIA VERDE COMO DESAFIOS TECNOLÓGICOS
}

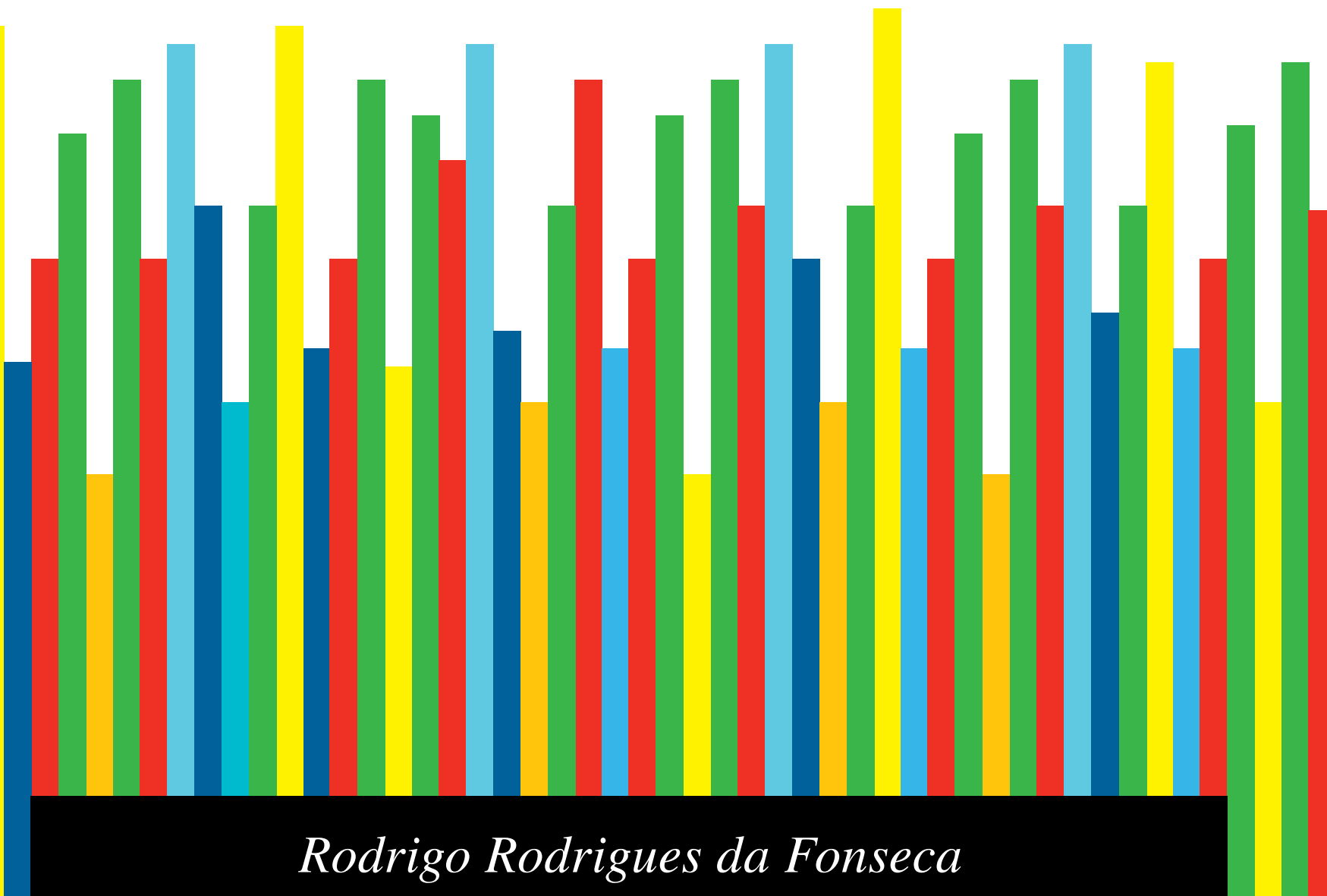


O artigo propõe reposicionar o debate ao abordar sustentabilidade como um desafio tecnológico. Para isso destaca a interconexão inerente entre a produção da tecnologia e a produção da sociedade, constatando a necessidade de políticas que possibilitem regimes de transição entre sistemas tecnológicos. Desse ponto parte o debate sobre o desafio específico brasileiro de encontrar uma matriz sustentável de desenvolvimento com redução das desigualdades sociais.

Palavras-chave: tecnologia, sustentabilidade, desenvolvimento, democracia.

\section{ABSTRACT}

This article aims at shifting the debate by approaching sustainability as a technological challenge. To do so it highlights the inherent connection between technology production and society production, and evinces the need of policies that allow for transitional arrangements between technological systems. A debate stems over the specific Brazilian challenge of finding a sustainable development matrix couple with the reduction of social inequality.

Keywords: technology, sustainability, development, democracy. 


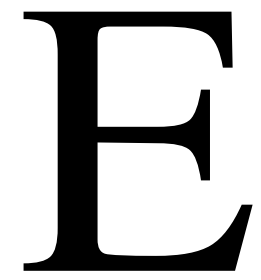

m primeiro lugar, é preciso avisar os leitores. Este texto não pretende convencer uma pessoa sequer do impacto da ação humana nas mudanças climáti-

cas. A premissa da qual partimos é de que isso é um dado e que há uma necessidade urgente de iniciar um processo contínuo e consistente de mudanças nos modos de produção e consumo que possibilitem uma sociedade global sustentável. Pretendemos discutir como ajudar a fortalecer, acelerar e sustentar esse processo, argumentando que ele ainda carece de consistência e apoio social porque a conexão entre sustentabilidade e tecnologia segue sendo formulada de forma inadequada.

Não formulamos a questão adequadamente porque muitas vezes não compreendemos os processos pelos quais a tecnologia surge, se desenvolve, ou não, e passa a fazer parte das nossas vidas interagindo com outros processos sociais para formar a base que possibilita a nossa existência cotidiana. Para produzir mudanças reais temos que mudar também a forma como fomentamos, desenvolvemos e usamos a tecnologia.

No convite para escrever este texto os editores da revista propuseram um interessante mote para quem trabalha em uma agência de fomento a ciência, tecnologia e inovação (CT\&I): "Os desafios tecnológicos da sustentabilidade e da economia verde". Se buscarmos na sustentabilidade alguns desafios tecnológicos, certamente vamos encontrar questões muito importantes. No entanto, cremos que, assim como definimos os problemas, vamos definir as soluções. Ao definir a sustentabilidade sobretudo como um problema tecnológico, trabalharemos para definir soluções em outro patamar de importância e partindo de conexões mais apuradas com o funcionamento da sociedade. Por isso, nos parece mais adequado formular o tema assim: "A sustentabilidade e a economia verde como desafios tecnológicos".

Não defendemos aqui que a sustentabilidade seja um problema exclusivamente tecnológico. Tecnologia não é apenas o artefato, mas também o sistema de conhecimentos e a organização necessária para produzi-la e operá-la (Thomas, 2009). A sustentabilidade é um problema da sociedade e, tal e qual, é um problema composto de dimensões econômicas, políticas, culturais, ecológicas, ideológicas, tecnológicas e de gênero.

A sociedade constrói a ciência e a tecnologia ao mesmo tempo em que a ciência e a tecnologia constroem a sociedade. Sem determinismos de parte a parte. Essa é, em geral, a lição mais difícil de compreender quando começamos a estudar as relações entre ciência, tecnologia e sociedade ${ }^{1}$. A construção da tecnologia é explicada segundo o jogo social no qual estão presentes atores com seus interesses, valores, com diferenças de poder, de saberes e de capacidades. Isso equivale a dizer que a tecnologia não segue um caminho predeterminado ou é sempre a "melhor" tecnologia. O sentido do desenvolvimento da tecnologia vai se dar de acordo com o complexo jogo de relações que se estabelecem em qualquer sistema social ${ }^{2}$.

A tecnologia predominante no mundo de hoje é a que inclui no seu desenvolvimento os valores e os interesses que predominam no jogo social e que servem para a construção desse tipo de sociedade. Se pensamos em uma sociedade com uma base material e tecnológica sustentável ou verde, temos de pensar em construir outro tipo de tecnologia. Isso porque, nesse jogo social, alguns dos "interesses" menos privilegiados ou negligenciados são os de sustentabilidade ambiental.

As preocupações com o desenvolvimento sustentável devem ir muito além dos efeitos do aquecimento global sobre as atividades humanas e tornam cada vez mais necessário o desenvolvimento de tecnologias que contribuam para a construção de uma economia mais verde.

\section{TRANSIÇÃO: PLANEJANDO OS CAMINHOS DA TECNOLOGIA}

Tratando a sustentabilidade como um desafio tecnológico, surge uma ampla agenda
RODRIGO RODRIGUES DA FONSECA é doutor em Política Científica e Tecnológica pela Unicamp e analista da Financiadora de Estudos e Projetos (Finep).

1 Nessa perspectiva não é possível considerar os artefatos como puramente tecnológicos ou puramente sociais, senão como resultados da dinâmica de proces sos deconstituição de "ensambles socio-téc nicos" (Bijker, 1995). Tanto a forma como o próprio funcionamento de um artefato se constituem como derivação contingente das disputas, pressões, resistências, negociações e convergências que vão conformando o conjunto heterogêneo de atores, conhecimentos e objetivos materiais.

2 Entre os principais estudos sociais da ciência e tecnologia podemos citar osclássicos estudos sobre a trajetória tecnológica da bicicleta e da geladeira de Pinch e Bjiker e estudos de outros autorescomoWinner Latour e Callon. 
de inovações. Desde pequenos avanços que reduzam a emissão de $\mathrm{CO}_{2}$, até mudanças em complexos sistemas tecnológicos como os de transporte ou de produção de energia. Interessante seria criar estratégias de fomento a regimes de transição tecnológica que ao mesmo tempo pressionassem e apoiassem mudanças.

Não é possível mudar subitamente a base material de suporte ao funcionamento da sociedade. Os grupos sociais não se dispõem a mudar hábitos de uso e consumo instantaneamente. Seriam necessárias várias etapas para alcançar um patamar satisfatório. Exemplos de mudanças de formas de consumo podem ser levantados. O caso do CFC é interessante de ser lembrado. Até os anos 80, o CFC era utilizado em vários produtos, em especial aerossóis, gases de refrigeração e produção de espuma. Após uma controvérsia que envolveu fabricantes, cientistas e órgãos reguladores de todo o mundo, a sociedade foi convencida de que o CFC era um dos principais causadores do buraco na camada de ozônio. Assim os fabricantes foram pressionados a encontrar outro produto que substituísse o CFC.

Nesse caso a pressão social, não a controvérsia científica, mudou uma trajetória tecnológica. Segundo dados da Organização Mundial de Meteorologia, a camada de ozônio deve se recompor perto de 2065. Os tempos de reação da sociedade e da natureza são distintos, mas, via de regra, demorados. Por isso, a complexidade e a persistência das políticas públicas são fatores-chave.

O desenvolvimento tecnológico pode ser "adequado" ao longo do tempo. Por isso, o financiamento público para ciência, tecnologia e inovação pode e deve induzir esse movimento de transição em todo o seu espectro, apoiando tanto as inovações incrementais que reduzem os danos causados por determinadas tecnologias, quanto as inovações mais radicais que vão substituir sistemas tecnológicos inteiros.

Um carro polui mais para ser produzido do que vai poluir em toda a sua vida útil. Portanto é necessário mudar a forma de produzir carros e também de locomoção. Não é possível mudar todas as formas de produção e uso de bens de forma súbita. Contudo, é possível fazê-lo de forma mais rápida e dirigida a objetivos, desde que esses sejam traçados e perseguidos. Parte significativa do sistema de locomoção de diversos países está baseada numa tecnologia que necessita de energia para mover uma tonelada para transportar, em média, uma pessoa de setenta quilos. Não é possível mudar em um ano o sistema de locomoção de uma cidade, muito menos de um país, devido aos limites econômicos, culturais, infraestruturais e tecnológicos. Mas é possível reduzir os impactos desse sistema; para isso necessitaremos de tecnologias que viabilizem isso sem desconfigurar o uso social do sistema tecnológico.

Tecnologias como o carro elétrico e os biocombustíveis adaptam-se ao sistema, reduzindo o custo ambiental da tecnologia. Em alguns anos é possível mudar o sistema de locomoção, exigindo outro aparato tecnológico que lhe dê sustentação. Para isso precisaremos de tecnologias que acompanhem a reconfiguração da infraestrutura e do uso social do sistema de locomoção.

A produção de energia é um dos principais temas no mundo quando se trata de sustentabilidade, em especial devido aos interesses econômicos e geopolíticos globais, aos montantes financeiros envolvidos e aos impactos no aquecimento global gerados pelos sistemas tecnológicos de produção e uso de energia. O Brasil ocupa posição de destaque na produção de energia a partir de fontes renováveis e de combustíveis alternativos detendo capacitações científicas e tecnológicas ligadas a sua matriz energética, que está entre as mais limpas do mundo. Nesse campo, o Brasil tem posição ambiental, econômica e tecnológica privilegiada. Mas ainda não está dado se o país vai mantê-la e se vai efetivamente tornar-se uma economia verde.

Independente de qualquer definição que se adote de economia verde, não podemos afirmar que esta já ocorre em algum país ou cidade. Alguns indicadores mostram que as ações que ligam economia e sustentabilida- 
de ainda têm poucos resultados. Analisando o desempenho histórico dos investimentos sustentáveis no mercado acionário brasileiro, Cunha (2011, p. 110) afirma que:

"Conclui-se que, embora haja evidências de um potencial promissor, os investimentos sustentáveis no mercado acionário brasileiro não obtiveram um desempenho financeiro satisfatório, indicando que as restrições ESG na alocação de recursos no país ainda não foram suficientes para afetar o ambiente econômico".

Transitar para uma economia verde e/ou de baixo carbono não parece ser uma tarefa fácil. Significa dirigir o desenvolvimento tecnológico através de parâmetros dados pelo conceito de sustentabilidade. Contudo, dizendo com todas as palavras, não é possível aplicar esses parâmetros na sua completude de imediato. Por isso é preciso construir a economia verde através de um sistema de transição que inclui mudança de atitude de empresas, governos e pessoas. Esse sistema pode ser fomentado por financiamentos e compras públicas, regulação, políticas públicas orientadas para sustentabilidade e conscientização social ampla. A política proposta pelo MCTI (2012, p. 37) dá passos interessantes nesse sentido:

"A economia verde, entendida como uma economia que promoverá o crescimento econômico, tendo como vetor central a vertente ambiental e a inclusão produtiva, pode ser a grande aposta estratégica brasileira. Para ser viável, deverá promover profunda transformação no setor produtivo: envolverá novos processos de produção e novos produtos e materiais recicláveis, os quais evitarão a utilização crescente de recursos naturais, dentre outros aspectos que serão necessariamente abordados para atender a hábitos de consumo de uma sociedade ambientalmente consciente. Ou seja, não basta apenas desenvolver tecnologia e inovação, elas têm de conduzir a um novo paradigma de produção e consumo. Essa é uma das vertentes centrais de onde pode provir o salto tecnológico brasileiro".
Por exemplo, $75 \%$ do tráfego mundial de pessoas e mercadorias passa por rodovias. Contudo, o usuário do transporte rodoviário paga no máximo $60 \%$ dos custos dessa infraestrutura. Ou seja, há um subsídio da sociedade para essa modalidade de transporte, que é sabidamente de maior impacto ambiental pelo uso predominante de combustíveis fósseis e menor eficiência energética por peso transportado. Não serão suficientes mudanças tecnológicas, serão imprescindíveis mudanças de distribuição de recursos para infraestruturas, legislação e financiamento.

Analisando o Brasil como uma "economia do conhecimento natural", Bound (2008) confirma o que já sabemos. A autora afirma que o sucesso do Proálcool brasileiro foi produzido por uma combinação de regulação, subsídios, impostos, apoio financeiro para expansão das destilarias, sistemas de distribuição e desenvolvimento tecnológico. Aí está outra evidência empírica. A sustentabilidade precisa começar a permear todas as ações públicas e privadas: compras públicas, financiamento ao desenvolvimento, infraestrutura, agricultura e políticas de CT\&I.

\section{POLÍTICA DE CT\&I: SUSTENTABILIDADE, INOVAÇÃO E FINANCIAMENTO PÚBLICO}

O discurso sobre sustentabilidade penetrou a área de CT\&I e os espaços de formulação de política. A 4⿳亠丷a Conferência Nacional de Ciência, Tecnologia e Inovação teve como mote principal o desenvolvimento sustentável. A consolidação das recomendações da $4^{\text {a }}$ Conferência de CT\&I traz um capítulo sobre a "Ampliação dos esforços de pesquisa em áreas e tecnologias estratégicas para o desenvolvimento sustentável brasileiro". A lista de esforços traz um mundo de setores e/ou áreas de conhecimento:

"Tecnologias ambientais e mitigação de mudanças climáticas; Biotecnologia e uso sustentável da biodiversidade; Recursos 
hídricos; Recursos minerais; Agricultura, pecuária, agropecuária e pesca; Tecnologias de uso e manejo do solo; Agronegócio; Agricultura familiar; Segurança alimentar; Agropecuária; Aquicultura e pesca; Saúde e fármacos; Espaço, defesa e segurança nacional; Sistemas urbanos sustentáveis e segurança pública; Fontes alternativas de energia e energias renováveis" (CGEE, 2010b).

Com o lançamento da Estratégia Nacional de Ciência, Tecnologia e Inovação (ENCTI), o MCTI avança bastante em comparação aos resultados da conferência. Passando da lista para uma estratégia, o ministério aponta os meios pelos quais pretende alcançar seus objetivos. A ENCTI afirma que, nos últimos anos, surgiu um conjunto amplo de novas tendências nas políticas de CT\&I nos países desenvolvidos e nos países em desenvolvimento. A primeira tendência destacada é a "preocupação com a sustentabilidade no seu sentido mais amplo, nas estratégias nacionais de pesquisa e de inovação. A maioria dos países desenvolvidos continua a colocar as questões ambientais, climáticas e de segurança energética no topo da agenda das estratégias nacionais de CT\&I" (MCTI, 2012, p. 37). As áreas de saúde e de qualidade de vida também se mantêm como prioridades importantes e estão bastante próximas à preocupação ambiental.

A ampliação das bases para a sustentabilidade ambiental passa pelo aprendizado do gestor público, em especial do financiador. Este deve ser capaz de construir a ponte entre as decisões da sociedade, expressas em escolhas políticas, e os setores que produzem e usam tecnologia. Essa ponte se constrói através do fomento público direcionado que induza agentes, em especial as empresas, a buscarem maneiras de produzir de forma mais sustentável. A sustentabilidade deve ser critério fundamental na pesquisa científica, no desenvolvimento de tecnologia, na formulação de políticas públicas e nos estudos sociais e/ou econômicos.

A sustentabilidade como desafio tecnológico precisa entrar nos cálculos dos engenheiros, cientistas e tecnólogos desde a formulação dos problemas, a escolha dos métodos de pesquisa, o desenho de artefatos, os métodos de cálculo de desempenho, o uso de insumos, o risco tecnológico, entre outros.

A sustentabilidade como desafio tecnológico precisa entrar nos cálculos dos economistas pela observação da trajetória tecnoprodutiva, da evolução econômica dos grupos sociais implicados, dos processos de aprendizagem e difusão e na evolução das relações socioeconômicas. Bem como precisam ter como referência em sustentabilidade os cálculos da estrutura de custos, de geração de preços, da reconfiguração de espaços de mercado, as dinâmicas de emprego, os mecanismos de financiamento e da própria sustentabilidade econômica das empresas, cidades, países, pessoas, etc.

A sustentabilidade como desafio tecnológico precisa entrar nos cálculos dos gestores públicos buscando a formulação, implementação e avaliação de políticas públicas, marcos normativos, estratégias de financiamento, infraestruturas e formação de quadros especializados, em particular, geração de instâncias de aprendizagem por interação e gestação de mecanismos de pesquisa e desenvolvimento e produção de tecnologias sustentáveis em rede que envolvam diferentes estratégias institucionais (unidades de $\mathrm{P} \& \mathrm{D}$, universidades, ONGs, agências governamentais, agências internacionais, empresas, etc.).

Um dos maiores desafios está na formação de quadros especializados com capacidades de elaboração de políticas para os diferentes níveis de tomada de decisões (local, municipal, estadual, nacional, internacional). Não basta apenas formar pós-graduados em grande quantidade. É preciso formá-los com critérios que incluam a sustentabilidade no jogo. É preciso preparar as pessoas e instituições para o ciclo de desenvolvimento que o país vem empreendendo.

\section{DESENVOLVIMENTO, SUSTENTABILIDADE E INOVAÇÃO}

Historicamente, o desenvolvimento brasileiro esteve baseado na incorporação do 


\section{QUADRO 1}

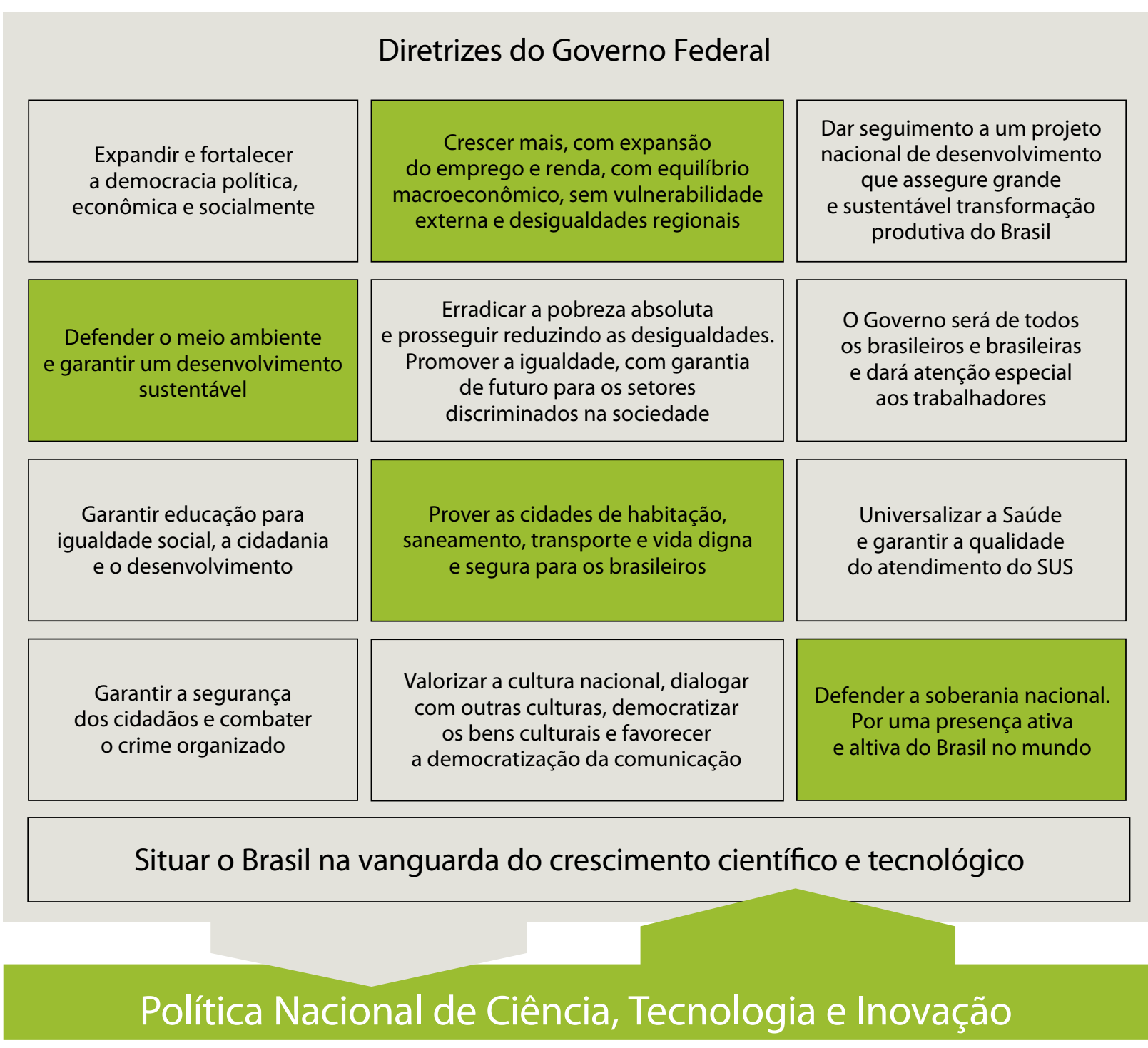

Fonte: MCTI, 2012, p. 29

progresso técnico à produção industrial e na utilização, via importação ou investimento estrangeiro direto, de equipamentos e tecnologias já disponíveis, em geral geradas em outros países. Contudo, hoje, não basta apoiar o avanço da industrialização baseado no desenvolvimento científico e tecnológico endógeno e em sua incorporação crescente ao processo produtivo. Mantido o modelo, apenas nos somaremos ao desastre promovido pelos países desenvolvidos.

O papel desempenhado pela CT\&I na competitividade entre nações não se limita à esfera econômica. A inovação e o conhecimento passaram a ser os motores do desenvolvimento. Isso impõe que a disseminação de novos conhecimentos, novas tecnologias e métodos seja capaz de ampliar o acesso a bens e serviços, gerando melhorias concretas e de qualidade de vida e reduzindo desigualdades sociais.

Porém, o ciclo de desenvolvimento iniciado nos anos 2000, baseado no crescimento com redistribuição de renda e na incorporação de grande contingente populacional ao mercado de consumo de massa, não tem 
preservado os aspectos essenciais da sustentabilidade. Reside aí o grande desafio nacional. A continuidade do atual ciclo de crescimento da economia brasileira e a construção de um novo padrão de desenvolvimento sustentável dão outro significado à política de CT\&I - a colocam em outro patamar de importância.

Como discurso e nos documentos, o atual governo tem ressaltado essa importância. Para o ex-ministro da CT\&I, Aloizio Mercadante:

"A prioridade, agora, é principalmente traduzir o desenvolvimento científico e tecnológico em progresso material e bem-estar social para o conjunto da população brasileira, o que passa pela convergência de dois macromovimentos estruturais: a revolução do sistema educacional e a incorporação sistemática ao processo produtivo, em seu sentido amplo, da inovação como mecanismo de reprodução e ampliação do potencial social e econômico do país. Esse é o caminho para transformar a ciência, a tecnologia e a inovação em eixo estruturante do desenvolvimento brasileiro" (MCTI, 2012, p. 10).

Essa prioridade também está refletida entre as treze diretrizes centrais do governo Dilma.

Lidas no sentido de tratar a sustentabilidade como desafio tecnológico, essas diretrizes oferecem uma vasta agenda de inovação com prioridades bem estabelecidas em cada um dos itens. Mais importante, as inovações realizadas poderiam ser viabilizadas pelo gasto público que já seria realizado de qualquer maneira. Faz parte do processo encontrar formas mais inteligentes e sustentáveis - através de organização

3 A visão de ciência e tecnologia como construção social foi amplamente estudada por diversos autores - como Callon, Pinch, Bjiker e Winner - ligados à corrente construtivista dentro dosestudos sociais da ciência e tecnologia. e tecnologia - de usar os escassos recursos do Estado. Apresenta-se assim uma agenda interessante para os Tribunais de Contas e Controladoria Geral da União.

Além disso, a própria ENCTI afirma que:

"É importante que o desenvolvimento brasileiro seja baseado: (i) na contenção e rever- são dos danos antrópicos associados aos padrões de produção e consumo da sociedade moderna; (ii) na adoção de novas modalidades de aproveitamento sustentável dos recursos naturais; (iii) na minimização do impacto ambiental dos processos de ocupação do território e de assentamento populacional, especialmente nas aglomerações urbanas e metropolitanas" (MCTI, 2012, p. 36).

É fato que a inovação terá papel fundamental no esforço de desenvolvimento sustentável. No entanto, para que seja efetiva a construção de uma sociedade justa e solidária e de um ambiente empresarial mais competitivo no plano internacional, a geração e apropriação do conhecimento científico e tecnológico deve buscar orientar-se por critérios até o momento negligenciados. Embora os discursos e documentos incluam avanços conceituais muito relevantes, transformá-los em realidade é em geral mais difícil do que escrevê-los.

Um modelo de desenvolvimento que tem como fim último o crescimento econômico organiza e subordina diversos campos da atividade aos seus propósitos e, em especial, à produção de ciência e tecnologia. Ocorre que esse modelo não dá conta da necessidade de geração de empregos, nem o planeta suporta o crescente nível de uso dos recursos naturais ou de geração de energia.

Estabelecer claramente essa relação entre ciência, tecnologia, inovação e desenvolvimento é fundamental para o tema que estamos discutindo. CT\&I não são campos de atividade fora dos sistemas de organização social $^{3}$. Assim como todos os campos de atividade humana, são construídas e, ao mesmo tempo, constroem esses sistemas de organização social. Portanto, CT\&I têm a sua parte de contribuição nessa forma de organização social que gera exclusão social e degradação ambiental.

Parte significativa da CT\&I produzida hoje está ligada ao modelo de desenvolvimento focado no crescimento econômico. É preciso unir forças capazes de introduzir na produção de CT\&I valores ligados ao modelo 
de desenvolvimento social e sustentável, que fará parte do processo de redução das desigualdades e aprofundamento da democracia no Brasil.

\section{TECNOLOGIA, SUSTENTABILIDADE, DEMOCRACIA E IGUALDADE SOCIAL}

O desafio é passar a trabalhar esses quatro conceitos em conjunto. Para alcançar o estilo de desenvolvimento de que estamos tratando é preciso instituir um processo de produção, uso e difusão do conhecimento que esteja conectado ao conceito de cidadania e inclua a participação crescente dos atores até agora dela excluídos. As políticas de Estado devem ser menos hierarquizadas e mais transparentes, possibilitando a participação efetiva e democrática daqueles atores. A consistência e o apoio social das políticas públicas e ações empresariais ligadas à sustentabilidade se viabilizarão pelo adequado tratamento da conexão entre sustentabilidade e tecnologia.

Passo essencial nessa direção é que os atores sociais envolvidos compreendam es- ses processos e incluam sustentabilidade nas suas agendas. Em especial cientistas, burocratas e integrantes de movimentos sociais precisam compreender como a mudança técnica impacta seus espaços de ação e como utilizar e apropriar-se do conhecimento e da tecnologia visando à transformação, evitando a reprodução da degradação ambiental e da exclusão social.

Muitas vezes o debate sobre sustentabilidade fica paralisado entre as posições dos que querem tudo e dos que não querem mudar nada. Não podemos ficar presos a essa armadilha, nem precisamos entrar no "caminho do meio". A falácia da terceira via soluciona temporariamente alguns conflitos, mas adia verdadeiras soluções. Um caminho de transição só será possível quando mudanças tecnológicas e sociais forem seriamente induzidas em etapas. Encarar o problema em todas as suas dimensões - econômicas, políticas, tecnológicas, ecológicas, culturais, regionais - é o primeiro passo para construir um caminho ainda não trilhado apoiado em trajetórias tecnológicas inovadoras e sustentáveis. Daqui para a frente o "como" nos desenvolvemos é mais importante do que o "quanto" crescemos.

BIJKER, W.; PINCH, T. “The Social Construction of Technological Systems: New Directions in the Sociology and History of Technology", in New Directions in the Sociology and History of Technology. London, MIT Press, 1987.

BOUND, K. Brazil: The Natural Knowledge Economy, the Atlas of Ideas. Londres, Demos, 2008.

CGEE. 4ª Conferência Nacional de Ciência, Tecnologia e Inovação para o

Desenvolvimento Sustentável. Brasília, 2010a. . Consolidação das Recomendações da 4a Conferência Nacional de Ciência, Tecnologia e Inovação para o Desenvolvimento Sustentável. Brasília, $2010 \mathrm{~b}$.

CUNHA, F. Análise de Desempenho dos Investimentos Sustentáveis no Mercado Acionário Brasileiro: um Estudo sobre o Índice de Sustentabilidade Empresarial (ISE). Dissertação de mestrado. Rio de Janeiro, Departamento de Engenharia Industrial, Pontifícia Universidade Católica do Rio de Janeiro, 2011. 
DE NEGRI, J.; LEMOS, M. (orgs.). O Núcleo Tecnológico da Indústria Brasileira. Brasília, Ipea-ABDI-Finep, 2011.

MCTI. Estratégia Nacional de Ciência, Tecnologia e Inovação (ENCTI). Brasília, 2012.

METCALFE, J. Stanley. "Equilibrium and Evolutionary Foundations of Competition and Technology Policy: New Perspectives on the Division of Labor and the Innovation Process", in Revista Brasileira de Inovação, volume 2, número 1. Rio de Janeiro, janeiro-junho, 2003.

NOBLE, David. America by Design: Science, Technology and the Rise of Corporate Capitalism. New York, Oxford University Press, 1977.

PINCH, T.; BIJKER, W. E. "The Social Construction of Facts and Artifacts: or How the Sociology of Science and Sociology of Technology Might Benefit Each Oder", in W. Bijker et al. The Social Construction of Technological Systems. Cambridge, MIT Press, 1987.

THOMAS, H. "Estructuras Cerradas vs. Procesos Dinámicos: Trayectorias y Estilos de Innovación Y Cambio Tecnológico", in H. A. Buch et al. Actos, Actores y Artefactos. Bernal, Universidad Nacional de Quilmes Editorial, 2007.

VEIGA, J. Mundo em Transe - do Aquecimento Global ao Ecodesenvolvimento. São Paulo, Armazém do Ipê, 2009.

WINNER, L. "Upon Opening the Black Box and Finding it Empty: Social Constructivism and the Philosophy of Technology", in Science, Technology, \& Human Values, 18, 1993, pp. 362-78. 